\title{
High Seebeck Coefficient and Power Factor in n- Type Organic Thermoelectrics
}

Guangzheng Zuo, Zhaojun Li, Ergang Wang and Martijn Kemerink

The self-archived postprint version of this journal article is available at Linköping University Institutional Repository (DiVA):

http://urn.kb.se/resolve?urn=urn:nbn:se:liu:diva-144566

N.B.: When citing this work, cite the original publication.

Zuo, G., Li, Z., Wang, E., Kemerink, M., (2018), High Seebeck Coefficient and Power Factor in n-Type Organic Thermoelectrics, ADVANCED ELECTRONIC MATERIALS, 4(1), 1700501.

https://doi.org/10.1002/aelm.201700501

Original publication available at:

https://doi.org/10.1002/aelm.201700501

Copyright: Wiley (12 months)

http://eu.wiley.com/WileyCDA/ 
DOI: $10.1002 /(($ please add manuscript number $))$

Article type: Communication

\section{High Seebeck coefficient and Power Factor in n-Type Organic Thermoelectrics}

Guangzheng Zuo, Zhaojun Li, Ergang Wang, and Martijn Kemerink*

\section{G. Zuo, Prof. M. Kemerink}

Complex Materials and Devices, Department of Physics, Chemistry and Biology (IFM), Linköping University, SE-58183, Linköping, Sweden

E-mail: martijn.kemerink@liu.se

Z. Li, Dr. E. Wang

Department of Chemistry and Chemical Engineering, Chalmers University of Technology, SE-41296, Göteborg, Sweden

Keywords: Seebeck coefficient; organic thermoelectrics; power factor; n-type doping

\section{Abstract:}

The n-type thermoelectric properties of PCBM are investigated for different solution-based doping methods. We introduce a novel inverse-sequential doping method where the semiconductor (PCBM) is deposited on a previously cast dopant (N-DPBI) film to achieve a very high power factor $\mathrm{PF} \approx 35 \mu \mathrm{W} \mathrm{m} \mathrm{m}^{-1} \cdot \mathrm{K}^{-2}$ with a conductivity $\sigma \approx 40 \mathrm{~S} / \mathrm{m}$. We show that also n-type organic semiconductors obey the $-1 / 4$ power law relation between Seebeck coefficient $S$ and $\sigma$ that was previously found for p-type materials. An analytical model on basis of variable range hopping unifies these results. The power law for n-type materials is shifted towards higher conductivities by 2 orders of magnitude w.r.t. that of p-type, suggesting strongly that n-type organic semiconductors can eventually become superior to their p-type counterparts. Adding a small fraction lower-LUMO material (NDI-CN) into PCBM leads to a higher $S$ for inverse-sequential doping but not for bulk doping due to different morphologies. 


\section{WILEY-VCH}

Thermoelectric generators (TEG) turn temperature differences, as e.g. generated by waste heat, directly to electricity. Organic thermoelectrics (OTE) are considered by many to be a promising candidate material system to be used in lower cost and higher efficiency energy conversion. ${ }^{[1]}$ An optimal TEG needs legs of both p-type and n-type thermoelectric materials that are connected electrically in series and thermally in parallel.

Recently, a lot of work has been reported on p-type thermoelectric organic semiconductors $(\mathrm{OSC})^{[2-6]}$, e.g. achieving a highest power factor PF over $300 \mu \mathrm{W} / \mathrm{m} \cdot \mathrm{K}^{-2}$ with a thermoelectric figure of merit $\mathrm{ZT} \approx 0.25$ based on PEDOT derivatives. ${ }^{[5]}$ Compared to p-type OTE, the development of n-type thermoelectric materials is lagging behind, which can be attributed to the absence of an n-type equivalent of PEDOT, the overall scarcity of n-type OSC and the inefficiency of most n-type dopants. Since most good OSC that are used as n-type materials like PCBM and N2200 have a LUMO around $-4.0 \mathrm{eV}^{[7,8]}$, it is very difficult to realize a stable dopant with a HOMO above $-4.0 \mathrm{eV}$ as would be required for efficient electron transfer. Fortunately, Wei et al. have reported a promising material, (4-(1,3- dimethyl-2,3-dihydro-1Hbenzoimidazol-2-yl)phenyl) dimethylamine (N-DMBI) that can be used as stable n-type dopant due to a two-step thermally activated doping mechanism. ${ }^{[9]}$ Very recently, Huang et al. used this dopant to achieve record values for $\mathrm{PF}=105 \mu \mathrm{W} / \mathrm{m} \cdot \mathrm{K}^{-2}$ and $\mathrm{ZT}=0.11$ at room temperature for the small molecule A-DCV-DPPTT. ${ }^{[10]}$ Some reports also have demonstrated that dihydro-1H-benzoimidazol-2-yl (N-DBI) derivatives can improve the electrical conductivity by several orders of magnitude in n-type OTE by solution-processing. E.g. Schlitz et al. have shown that solution mixtures of P(NDIOD-T2) with 9 mol.\% N-DBI derivatives can achieve $\sigma \approx 1 \mathrm{~S} / \mathrm{m}$ and a PF over $0.6 \mu \mathrm{W} / \mathrm{m} \cdot \mathrm{K}^{-2} \cdot{ }^{[1]}$ Yuan et al. reported a small-molecule 2DQTT-o-OD that, with $10 \mathrm{wt} . \%$ of a novel N-DBI derivative incorporated in solution, acquires a PF of $17.2 \mu \mathrm{W} / \mathrm{m} \cdot \mathrm{K}^{-2}$ at room temperature. ${ }^{[12]}$ Shi et al. achieved a high electron mobility in FBDPPV with $\sigma \approx 1400 \mathrm{~S} / \mathrm{m}$ and $\mathrm{PF} \approx 28 \mu \mathrm{W} / \mathrm{m} \cdot \mathrm{K}^{-2}$, when adding around 5 wt.\% N-DMBI in solution. ${ }^{[13]}$ Despite the positive effect of N-DMBI and N-DBI 


\section{WILEY-VCH}

derivatives on electrical conductivity, it probably fails to further increase $\sigma$ and PF upon further increasing the volume fraction in solution mixtures due to degradation of the blend morphology. ${ }^{[14]}$ Liu et al. demonstrated a modified fullerene derivative, PTEG-1, that shows an increased miscibility at the nanoscale level and thereby achieved a PF of $16.7 \mu \mathrm{W} / \mathrm{m} \cdot \mathrm{K}^{-2}$ with $\sigma \approx 205 \mathrm{~S} / \mathrm{m}$ at $40 \mathrm{~mol} . \%$ of N-DMBI. ${ }^{[15]}$

For p-type OTE, most reported experimental data seem to follow an empirical quasi-universal power law relationship between the Seebeck coefficient $S$ and conductivity as $S \propto \sigma^{-1 / 4}$. ${ }^{[2,3]}$ In view of the limited number of reported data, it is still unclear whether n-type OTE also follow this power law, and different power law slopes have been reported. ${ }^{[15,16]}$ Here, we introduce a novel, inverse-sequential doping procedure that mitigates the need for solvent orthogonality in conventional sequential doping to investigate the potential of n-type OTE by studying the most common n-type OSC, PCBM, doped with an N-DBI derivative. We obtain a very high n-type $\mathrm{PF} \approx 35 \mu \mathrm{W} / \mathrm{m} \cdot \mathrm{K}^{-2}$, with a $\sigma \approx 40 \mathrm{~S} / \mathrm{m}$ using inverse-sequential doping vs. $\mathrm{PF} \approx 3 \mu \mathrm{W} / \mathrm{m} \cdot \mathrm{K}^{-2}$ and $\sigma \approx 6 \mathrm{~S} / \mathrm{m}$ using conventional bulk doping. We unify the behavior of n-type and p-type solution processed OTE by demonstrating that also n-type OTE follow the $-1 / 4$ power law relationship, which is important for the further development of (ntype) OTE as it sets stringent boundary conditions to the attainable PF and ZT as will be shown below. Surprisingly, for n-type OTE the quasi-universal power law is shifted to higher conductivities by around 2 orders of magnitude as compared to p-type, which we rationalize in terms of a higher (effective) attempt-to-hop frequency. As such, n-type OSC might have a potential to achieve high ZT than p-type OSC. In particular, our model indicates that ZT $\rightarrow 1$ should be a realistic target for n-type OTE.

The materials in this study are displayed in Figure 1 along with their relevant energy levels. PCBM is selected as a well-studied model system with a relatively high electron mobility that is readily doped by N-DPBI upon mild annealing, cf. the PCBM LUMO and the N-DPBI singly occupied molecular orbital (SOMO) levels - the latter is formed upon deprotonation 


\section{WILEY-VCH}

during annealing. ${ }^{[13]}$ To overcome the drawbacks of bulk doping, in which the semiconductor and the dopant are mixed in a common solution which tends to compromise morphology and thereby conductivity, ${ }^{[6,14]}$ we used a simple solution-based method by depositing the PCBM film on a previously cast dopant (N-DPBI) film. We developed the 'inverse-sequential doping' method since normal sequential doping led to the PCBM film dissolving in the NDPBI solution. ${ }^{[14]}$ For comparison, we also fabricated devices using conventional bulk doping. Further details are given in the experimental section.

The electrical conductivity and Seebeck coefficient have been investigated and the results are shown in Figure 2. For pure PCBM bulk doped with 5 wt.\% N-DPBI in solution, we achieve a conductivity $\sigma \sim 6 \mathrm{~S} / \mathrm{m}$, and a corresponding $S \sim 730 \mu \mathrm{V} / \mathrm{K}$. For inverse-sequential doping, we find a conductivity over $40 \mathrm{~S} / \mathrm{m}$ and $S \sim 860 \mu \mathrm{V} / \mathrm{K}$. Hence, by depositing the PCBM film on pre-cast N-DPBI film, the method of inverse sequential surface doping can achieve significantly higher conductivities than bulk doping; the change in thermopower is comparable to the sample-to-sample variation as shown by the error bars.

Adding significant amounts of heterogeneous 'guest' species to a common casting solution, as done in bulk doping, is known to compromise the morphology of host compounds that are well ordered in their pure state. ${ }^{[14]}$ We therefore attribute the increased conductivity, and virtually unaltered thermopower, of the inverse-sequentially doped samples to a better morphology that effectively leads to faster transport while the energetics, that determine the thermopower, are largely unaffected. ${ }^{[6,17]}$ In terms of the simple model that is discussed below, the effect of the improved morphology would be mapped on the (increased) attempt to hop frequency, which increases conductivity while leaving the thermopower unaffected. The mentioned values combine into a maximum $\mathrm{PF} \approx 35 \mu \mathrm{W} / \mathrm{m} \cdot \mathrm{K}^{-2}$ (average $\mathrm{PF}, 30 \pm 5 \mu \mathrm{W} / \mathrm{m} \cdot \mathrm{K}^{-}$ ${ }^{2}$ ), which is among the highest n-type power factor that has been reported for solution processed OTE. 


\section{WILEY-VCH}

In our previous work we have shown that density of states (DOS) engineering can be used to boost the thermopower of p-type OTE. ${ }^{[18]}$ This is relevant to low-power, low-cost systems, where voltage and price are more important than power, e.g. in thermometry or to supply an autonomous sensor or a reflective LCD display with electricity. ${ }^{[18-20]}$ Specifically, we add lower-LUMO material, NDI-CN ${ }^{[21]}$, into the PCBM solution, causing the formation of a second 'trap' maximum in the electron density of states, cf. Figure 1. Since the LUMO of PCBM sits at about $-4.0 \mathrm{eV}$ and the LUMO of NDI-CN at $-4.6 \mathrm{eV}$, we estimate a trap state that in this case sits some $0.6 \mathrm{eV}$ below the DOS maximum that is associated with PCBM. ${ }^{[21]}$ When energetics and concentrations are properly optimized, this causes the Fermi level to sit on the guest-part of the DOS whereas the transport energy remains on the host-part. This leads to a strong increase in $S$, albeit at the cost of a lower conductivity. ${ }^{[18]}$ Indeed, we observe an overall decrease in conductivity with increasing NDI-CN fraction in Figure $2 \mathrm{a}, \mathrm{b}$ that is accompanied by an obvious peak in $S$ for inverse-sequential doping at $1 \mathrm{wt} \%$ NDI-CN. Surprisingly, a negligible increase in $S$ occurs at the same concentrations for bulk doping. The highest observed $S \approx 1400 \mu \mathrm{V} / \mathrm{K}$ (average $S=1318 \pm 90 \mu \mathrm{V} / \mathrm{K}$ ) corresponding to a $\mathrm{PF} \approx 5$ $\mu \mathrm{W} / \mathrm{m} \cdot \mathrm{K}^{-2}$ as shown in Figure $2 \mathrm{~b}$.

To identify a possible cause for the pronounced difference in thermopower between inversesequential and bulk doping for PCBM:NDI-CN blends we inspected the film morphology by atomic force microscopy (AFM) as shown in Figure 3. We found that the morphology of PCBM:NDI-CN became distinctively different, depending on the NDI-CN concentration and the doping method, as also illustrated in the bottom insets of Figure 2. Figure 3 shows a pronounced phase separation beyond $1 \mathrm{wt} . \%$ NDI-CN in the case of reverse-sequential doping, whereas the phase separation is suppressed, i.e. converted into a smooth morphology, when 5 wt.\% N-DPBI is added to the PCBM:NDI-CN solution as bulk doping. Although the peak in $S$ occurs at 1 wt.\% NDI-CN, at which neither the reverse-sequential nor the bulk doped film shows signs of phase separation, this observation highlights, again, the need for 


\section{WILEY-VCH}

accurate morphology control. In contrast to the case of a single doped OSC, where quantitative differences in predominantly the conductivity where found for different morphologies, we find qualitative differences for our blended OSC. We speculate that different degrees of NDI-CN aggregation, possibly in combination with associated shifts in energy levels, are the reason for the observed differences. We reiterate that NDI-CN is not acting as dopant but as DOS-modifying agent and that the mechanism by which it affects conductivity and thermopower is therefore a fundamentally different one than for dopants. The connection of the above with morphology will be topic of future work.

To investigate the power law for both p- and n-type OSC, we developed a simple analytical model based on variable range hopping in a Gaussian disordered OSC. ${ }^{[22,23]}$ The thermopower $S$ is then to good approximation given by $S=\left(E_{F}-E_{t r}\right) / T$ with $E_{F}$ the Fermi energy, $E_{t r}$ the transport energy and $T$ the temperature. The model was used to calculate both thermopower and conductivity as a function of relative (to the total DOS) doping concentration $c\left(c=1 \times 10^{-6}-1 \times 10^{-1}\right)$. The results are plotted as thermopower vs. conductivity parametric in the attempt to hop frequency $\left(v_{0}\right)$ ranging from $5 \times 10^{10} \mathrm{~s}^{-1}$ to $5 \times 10^{13} \mathrm{~s}^{-1}$ (solid lines), and compared to the experimental data (dots) in Figure 4. All gray dots for p-type data and the dark yellow dots for n-type data have been measured in our group. We found that most of the p-type data are well described by the calculations when using a $v_{0}$ in the range $5 \times 10^{10} \mathrm{~s}^{-1}-5 \times 10^{11} \mathrm{~s}^{-1}$. Interestingly, for n-type data good agreement is found with the same model parameters except for a $v_{0}$ that is two orders of magnitude higher, i.e. $5 \times 10^{12} \mathrm{~s}^{-1}-$ $5 \times 10^{13} \mathrm{~s}^{-1}$, with the experimental data from inverse-sequential doping (dark yellow dots with red circle) sitting at higher $v_{0}$ than those of bulk doping. We note that the n-type data from Ref. ${ }^{[16]}$ are an exception to this rule and coincide with typical p-type data, see SI Figure S1. As a deviating dopant (TDAE) is used in this work and one of the polymers used, N2200 or $\mathrm{P}\left(\right.$ NDI2OD-T2), also appears in the data following the $v_{0}=5 \times 10^{12} \mathrm{~s}^{-1}$ model curve, we 


\section{WILEY-VCH}

attribute this exception to (morphological) problems associated with the particular dopant TDAE.

Hence, also the n-type OTE seem to follow the quasi-universal -1/4 power law relationship, but shifted to higher conductivities by around 2 orders of magnitude. A graph with more experimental data from literature embedded is shown in the Supporting Information (SI). In view of the general perception of n-type OSC performance being less than that of p-type, the data in Figure 4 may seem surprising.

The physical meaning of the key parameter $v_{0}$ is that of an attempt to hop frequency, which in the used Miller-Abrahams expression for the hopping rate is equal to the rate of energetically downward hops. In view of the very simplified nature of the model, in which e.g. morphological factors are only implicitly accounted for (in the values of the input parameters), the values for $v_{0}$ presented here should be regarded as effective numbers only. Nevertheless, we note that the observed higher attempt to hop frequency for electrons is entirely consistent with recent results from kinetic simulations of organic solar cells. Melianas et al. found $v_{0}$ around $1 \times 10^{13} \mathrm{~s}^{-1}$ for electron transport in PCBM and N2200, whereas $v_{0}$ around $1 \times 10^{10} \mathrm{~s}^{-1}-1 \times 10^{11} \mathrm{~s}^{-1}$ was found for hole transport in a range of polymer OSC. ${ }^{[24,25]}$ Hence, irrespective of the precise meaning of $v_{0}$, the implication of the findings in Figure 4 is that n-type OSC might eventually become superior to their p-type counterpart if one succeeds to reach the end of the model curves in Figure 4, i.e. reach a relative doping concentration $\sim 10 \%$ without compromising morphology.

To estimate the application potential for $n$ - and p-type OSC in organic thermoelectric generators, we made contour plots of $S$ vs. $\sigma$ for constant ZT from 0.001 to 1 , following the definition of $Z T=\frac{\sigma S^{2} T}{\kappa}$. Here, the thermal conductivity $\kappa=\kappa_{e}+\kappa_{l}$, with $\kappa_{e}$ the electronic contribution to $\kappa$ that is described by the Wiedemann-Franz law as $\kappa_{e}=\left(k_{B} / q\right)^{2} L \sigma T$, where $k_{B}$ is the Boltzmann constant, $q$ the elementary charge, and $L$ the dimensionless Lorenz factor; 


\section{WILEY-VCH}

For simplicity, we take $L=L_{0}$, with $L_{0}=\pi^{2} / 3$ the theoretical Sommerfeld value for a degenerate Fermi gas. ${ }^{[26]}$ The lattice thermal conductivity $\kappa_{l}$ was taken constant at $0.2 \mathrm{~W} \mathrm{~m}^{-1}$ $\mathrm{K}^{-1}{ }^{[27]}$ Figure $\mathrm{S} 2$ shows $\kappa$ vs. $\sigma$. The resulting plots of $S$ vs. $\sigma$ are shown in Figure 5 as dashed lines; the solid lines represent the calculated results from Figure 4. The data in Figure 5 indicate that typical chemically doped p-type OTE, for which the thermoelectric data sit around or below curves with $v_{0}=5 \times 10^{11} \mathrm{~s}^{-1}$ (Figure 4 and S1), have a low potential for use in actual TEG as witnessed by a ZT $<0.01$. The notable exception is (oxidatively doped) PEDOT-Tos, as shown by the gray dot in Figure $5 .{ }^{[5]}$ Note that the measured ZT $\approx 0.25$ is in good agreement with the estimate on basis of the contour lines in Figure 5.

For n-type OTE, most data for bulk doping sit around curves with $v_{0} \approx 5 \times 10^{12} \mathrm{~s}^{-1}$ (Figure 4), which can reach $\mathrm{ZT} \leq 0.1$ according to Figure 5. The conductivity and thermopower reported for N-DMBI-doped A-DCV-DPPTT in Ref.[10] land the dark red dot just above ZT = 0.1, in excellent agreement with the reported value of 0.11 . For inverse-sequential doping, ZT $\geq 0.1$ seems completely reachable. For the best data in this work, the light and dark yellow dots, we estimate $\mathrm{ZT} \approx 0.01$ and $\mathrm{ZT} \approx 0.07$, respectively. Extrapolation along the curves with $v_{0}=$ $5 \times 10^{13} \mathrm{~s}^{-1}$ or even $5 \times 10^{14} \mathrm{~s}^{-1}$ suggests that $\mathrm{ZT} \approx 0.3$ or even $\mathrm{ZT} \approx 1$ is reachable for $\mathrm{n}$-type organic thermoelectrics at room temperature.

In conclusion, we developed a simple solution-based inverse-sequential doping method to achieve high conductivity while preserving a high Seebeck coefficient for the prototypical ntype organic semiconductor PCBM, and obtained a very high power factor $\mathrm{PF} \approx 35 \mu \mathrm{W} / \mathrm{m}^{-} \mathrm{K}^{-}$ ${ }^{2}$. Although this was not explicitly investigated, the inverse-sequential doping method should be rather generally applicable and loosen the constraints in terms of solvent orthogonality on material systems to be used in low-cost all-solution based device fabrication. With 1 wt.\% of NDI-CN added into the N-DPBI doped PCBM, we achieved a highest $S$ around $1400 \mathrm{~S} / \mathrm{m}$ while maintaining a $\mathrm{PF} \approx 5 \mu \mathrm{W} / \mathrm{m} \cdot \mathrm{K}^{-2}$, confirming the general applicability of this facile 


\section{WILEY-VCH}

method to enhance $S$. A simple analytical model was used to unify the thermopower and conductivity of both p- and n-type OTE, and we found good consistency between the experimental data and our calculated curves. We found that most p-type data are described well by the model using an attempt to hop frequency $v_{0}$ around $0.5-5 \times 10^{11} \mathrm{~s}^{-1}$, whereas $v_{0}$ around $0.5-5 \times 10^{13} \mathrm{~s}^{-1}$ is required for n-type data. The implication of this finding is that n-type organic semiconductors seem to have more potential to eventually achieve high thermoelectric figures of merit $\mathrm{ZT} \rightarrow 1$ than $\mathrm{p}$-type materials. 


\section{WILEY-VCH}

\section{Experimental Section}

Materials: [6,6]-Phenyl- $\mathrm{C}_{61}$-butyric acid methyl ester (PCBM) was purchased from 1-

Material Inc. 4-(1,3-Dimethyl-2,3-dihydro-1H-benzoimidazol-2-yl)-N, N-diphenylaniline (NDPBI) was purchased from Sigma-Aldrich. Core-cyanated naphthalene diimide (NDI-CN) was synthesized at Chalmers University of Technology.

Device fabrication: a). Bulk doping: All materials were dissolved in ortho-dichlorobenzene (o-DCB). PCBM in a solution of $30 \mathrm{mg} / \mathrm{ml}$; NDI-CN in solutions of 5,10 and $20 \mathrm{mg} / \mathrm{ml}$; NDPBI in a solution of $5 \mathrm{mg} / \mathrm{ml}$. PCBM and NDI-CN solutions were taken by volume to form mixtures with an NDI-CN fraction from $1 \%$ to $20 \%$, and then $5 \mathrm{wt} . \%$ N-DPBI was added by volume to form the final casting solutions. The active layers were spin-coated ( $500 \mathrm{rpm}$ for 60 seconds and then $3000 \mathrm{rpm}$ for 20 seconds) on top of cleaned glass substrates to get thin films with a thickness around $30 \mathrm{~nm}$ as measured with a Dektak surface profilometer. $b$ ). Inversesequential doping: PCBM with $x \%$ NDI-CN solutions were made as above $(1 \leq x \leq 20)$. NDPBI was dissolved in a mixture of tetrahydrofuran (THF) and dichloromethane (DCM) in a 4:1 ratio and a concentration of $20 \mathrm{mg} / \mathrm{ml}$. The N-DPBI layer was firstly spin-coated on cleaned glass substrates and then the PCBM with $x \%$ NDI-CN layer was spin-coated on top of the N-DPBI layer. After that $\mathrm{LiF}(0.6 \mathrm{~nm})$ and $\mathrm{Al}(100 \mathrm{~nm})$ were thermally evaporated through a shadow mask under a pressure of $1 \times 10^{-6}$ mbar to form Ohmic contacts. The contact dimensions are $2 \times 7 \mathrm{~mm}$ (width $\times$ length), defining a $7 \times 0.5 \mathrm{~mm}$ channel. All samples were thermally annealed at $120{ }^{\circ} \mathrm{C}$ for 20 mins immediately after deposition of the top contact in an $\mathrm{N}_{2}$-filled glove box.

Conductivity and Seebeck coefficient measurement: Room temperature electrical characterization was performed in a glove box under Nitrogen atmosphere. Current voltage characteristics were obtained between $-50 \mathrm{mV}$ and $50 \mathrm{mV}$. Conductivities were calculated according to $\sigma=j / F$, where $j$ and $F$ are the current density and electric field, respectively. The thermopower was obtained by applying a linear temperature gradient of various magnitudes 


\section{WILEY-VCH}

along the sample and recording the shift of the $j V$-characteristics at the end of a settling time of ca. 400 seconds. From the change of the thermovoltage $\Delta V$ as a function of the temperature difference $\Delta T$ between the contacts, the thermopower $S$ was calculated as $S=\Delta V / \Delta T$. All electrical measurements have been done in a Kelvin-probe (4-probe) setup.

Sample stability: fabricated samples were found to have a stable power factor on the time scale of at least a week when kept under glove box conditions. Exposure to ambient air and light leads to a loss in conductivity by 1-2 orders of magnitude in a few hours.

Atomic force microscope (AFM) measurement: AFM images were measured by a Veeco Dimension 3100 with the films prepared as above.

\section{Supporting Information}

Supporting Information is available from the Wiley Online Library or from the author.

\section{Acknowledgements}

The research by G.Z. is supported by the Chinese Scholarship Council (CSC). We are grateful to Hassan Abdallah for technical assistance with the thermoelectric measurements, and Olle Inganäs for the use of technical infrastructure.

\section{References}

[1] O. Bubnova, X. Crispin, Energy Environ. Sci. 2012, 5, 9345.

[2] B. Russ, A. Glaudell, J. J. Urban, M. L. Chabinyc, R. A. Segalman, Nat. Rev. Mater. 2016, 1 , natrevmats 201650.

[3] A. M. Glaudell, J. E. Cochran, S. N. Patel, M. L. Chabinyc, Adv. Energy Mater. 2015, 5, 1401072.

[4] Q. Zhang, Y. Sun, W. Xu, D. Zhu, Adv. Mater. 2014, 26, 6829.

[5] O. Bubnova, Z. U. Khan, A. Malti, S. Braun, M. Fahlman, M. Berggren, X. Crispin, Nat. Mater. 2011, 10, 429.

[6] S. N. Patel, A. M. Glaudell, K. A. Peterson, E. M. Thomas, K. A. O’Hara, E. Lim, M. L. Chabinyc, Sci. Adv. 2017, 3, e1700434.

[7] S. H. Yoo, J. M. Kum, S. O. Cho, Nanoscale Res. Lett. 2011, 6, 545.

[8] J. W. Jung, J. W. Jo, C.-C. Chueh, F. Liu, W. H. Jo, T. P. Russell, A. K.-Y. Jen, Adv. Mater. 2015, 27, 3310.

[9] P. Wei, J. H. Oh, G. Dong, Z. Bao, J. Am. Chem. Soc. 2010, 132, 8852. 


\section{WILEY-VCH}

[10] D. Huang, H. Yao, Y. Cui, Y. Zou, F. Zhang, C. Wang, H. Shen, W. Jin, J. Zhu, Y. Diao, W. Xu, C. Di, D. Zhu, J. Am. Chem. Soc. 2017, 139, 13013.

[11] R. A. Schlitz, F. G. Brunetti, A. M. Glaudell, P. L. Miller, M. A. Brady, C. J. Takacs, C. J. Hawker, M. L. Chabinyc, Adv. Mater. 2014, 26, 2825.

[12] D. Yuan, D. Huang, C. Zhang, Y. Zou, C. Di, X. Zhu, D. Zhu, ACS Appl. Mater. Interfaces 2017, 9, 28795.

[13] K. Shi, F. Zhang, C.-A. Di, T.-W. Yan, Y. Zou, X. Zhou, D. Zhu, J.-Y. Wang, J. Pei, J. Am. Chem. Soc. 2015, 137, 6979.

[14] D. T. Scholes, S. A. Hawks, P. Y. Yee, H. Wu, J. R. Lindemuth, S. H. Tolbert, B. J. Schwartz, J. Phys. Chem. Lett. 2015, 6, 4786.

[15] J. Liu, L. Qiu, G. Portale, M. Koopmans, G. ten Brink, J. C. Hummelen, L. J. A. Koster, Adv. Mater. 2017, 29, 1701641.

[16] S. Wang, H. Sun, U. Ail, M. Vagin, P. O. Å. Persson, J. W. Andreasen, W. Thiel, M. Berggren, X. Crispin, D. Fazzi, S. Fabiano, Adv. Mater. 2016, 28, 10764.

[17] S. van Reenen, M. Scheepers, K. van de Ruit, D. Bollen, M. Kemerink, Org. Electron. 2014, $15,3710$.

[18] G. Zuo, X. Liu, M. Fahlman, M. Kemerink, Adv. Funct. Mater. 2017, 1703280.

[19] D. Zhao, S. Fabiano, M. Berggren, X. Crispin, Nat. Commun. 2017, 8, ncomms 14214.

[20] D. Zhao, H. Wang, Z. U. Khan, J. C. Chen, R. Gabrielsson, M. P. Jonsson, M. Berggren, X. Crispin, Energy Environ. Sci. 2016, 9, 1450.

[21] G. Zuo, Z. Li, O. Andersson, H. Abdalla, E. Wang, M. Kemerink, J. Phys. Chem. C 2017, 121, 7767.

[22] G. Zuo, H. Abdalla, M. Kemerink, Phys. Rev. B 2016, 93, 235203.

[23] H. Abdalla, G. Zuo, M. Kemerink, unpublished n.d.

[24] A. Melianas, V. Pranculis, Y. Xia, N. Felekidis, O. Inganäs, V. Gulbinas, M. Kemerink, Adv. Energy Mater. 2017, 1602143.

[25] A. Melianas, F. Etzold, T. J. Savenije, F. Laquai, O. Inganäs, M. Kemerink, Nat. Commun. 2015, 6, 8778.

[26] A. Weathers, Z. U. Khan, R. Brooke, D. Evans, M. T. Pettes, J. W. Andreasen, X. Crispin, L. Shi, Adv. Mater. 2015, 27, 2101.

[27] S. D. Kang, G. J. Snyder, Nat. Mater. 2017, 16, 252.

[28] T. Menke, P. Wei, D. Ray, H. Kleemann, B. D. Naab, Z. Bao, K. Leo, M. Riede, Org. Electron. 2012, 13, 3319. 
WILEY-VCH

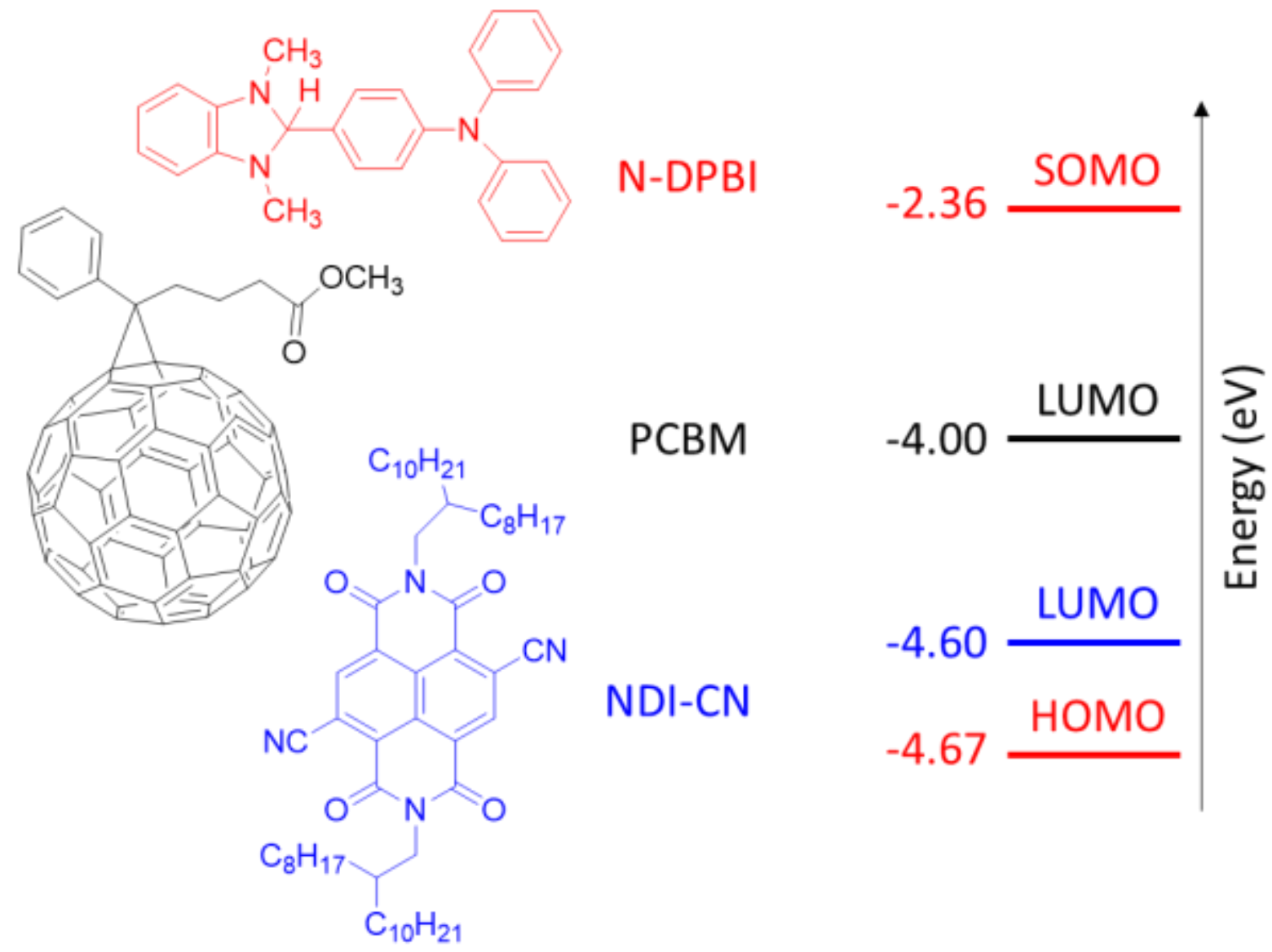

Figure 1 Chemical structure and relevant energy levels of the used materials.
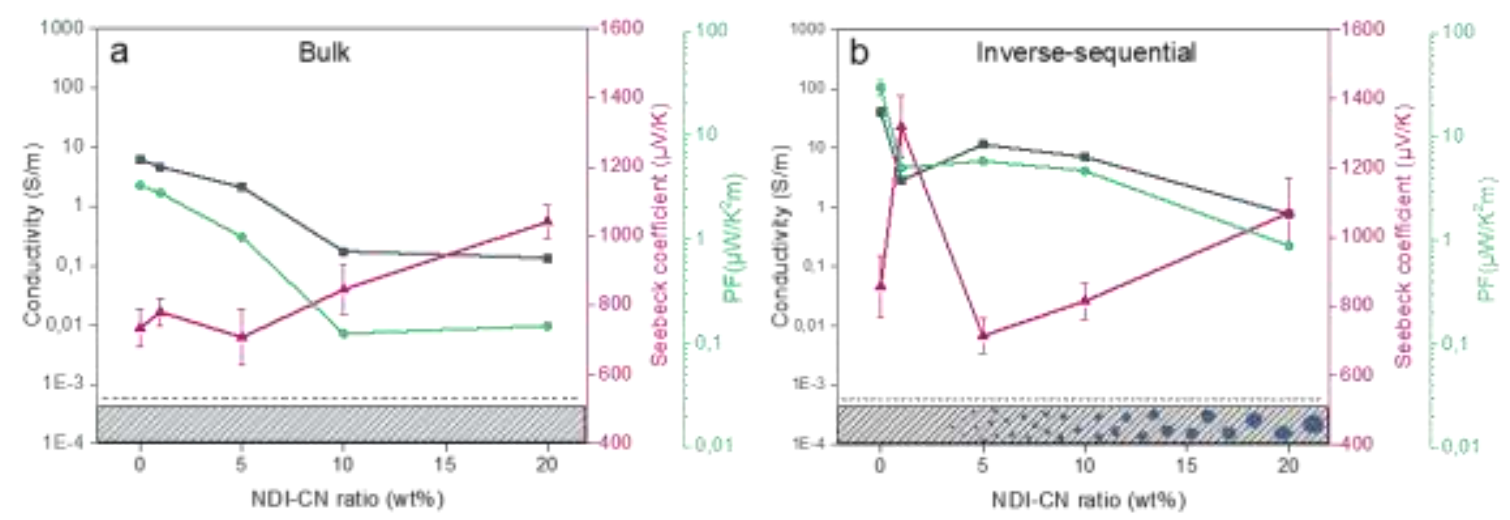

Figure 2 Conductivity, Seebeck coefficient and power factor (PF) vs. NDI-CN guest concentration for (a) bulk doping with $5 \mathrm{wt} . \% \mathrm{~N}-\mathrm{DPBI}$ and (b) inverse-sequential doping. The dashed line is the conductivity of pure PCBM without N-DPBI. Bottom inset: schematic morphology for the ternary system as extracted from Figure 3. Data points and error bars are obtained by averaging over at least 5 samples made at different times. 


\section{WILEY-VCH}

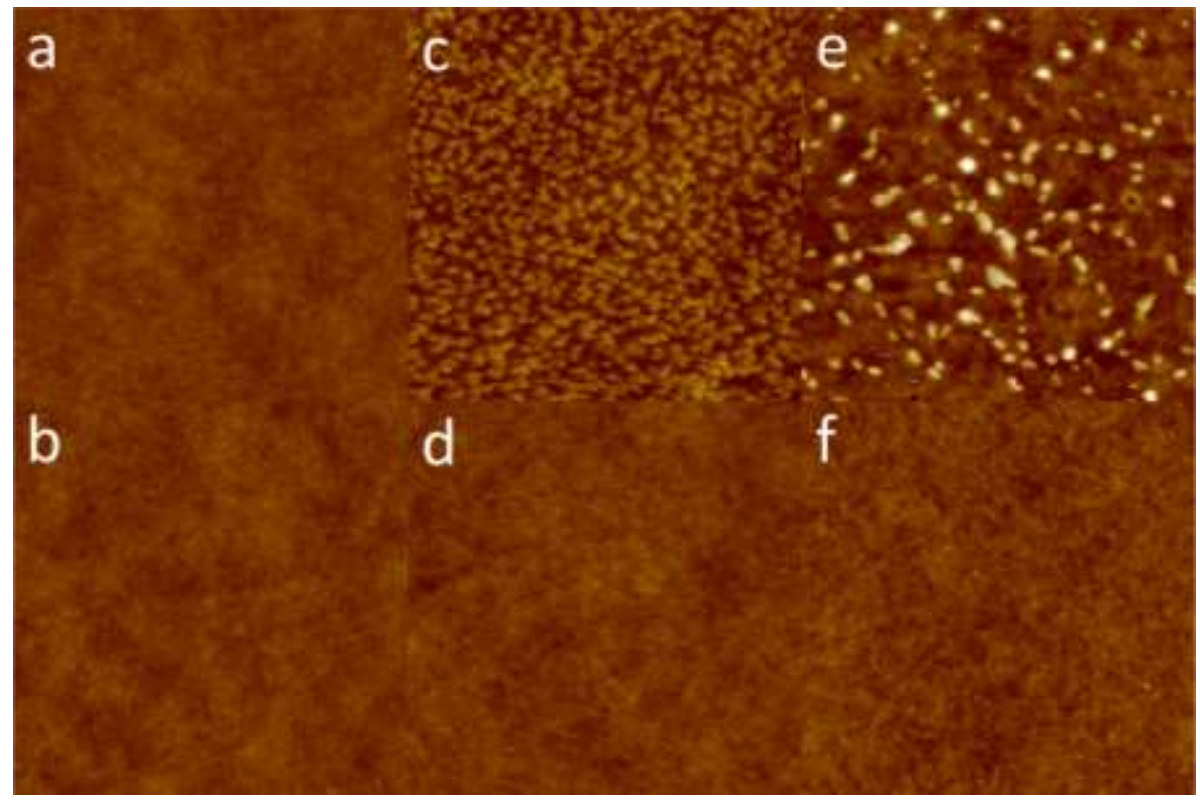

Figure 3 AFM height images for (a) pure PCBM; (b) PCBM:NDI-CN 1 wt\%; (c,d)

PCBM:NDI-CN 5\%; (e,f) PCBM:NDI-CN 10 wt\%. Samples d, f are bulk doping with 5 wt.\% $\mathrm{N}$-DPBI; $\mathrm{a}, \mathrm{b}, \mathrm{c}$, e are inverse-sequential doping. Scan size is $2 \times 2 \mu \mathrm{m}$, vertical scale is $10 \mathrm{~nm}$ in all images.

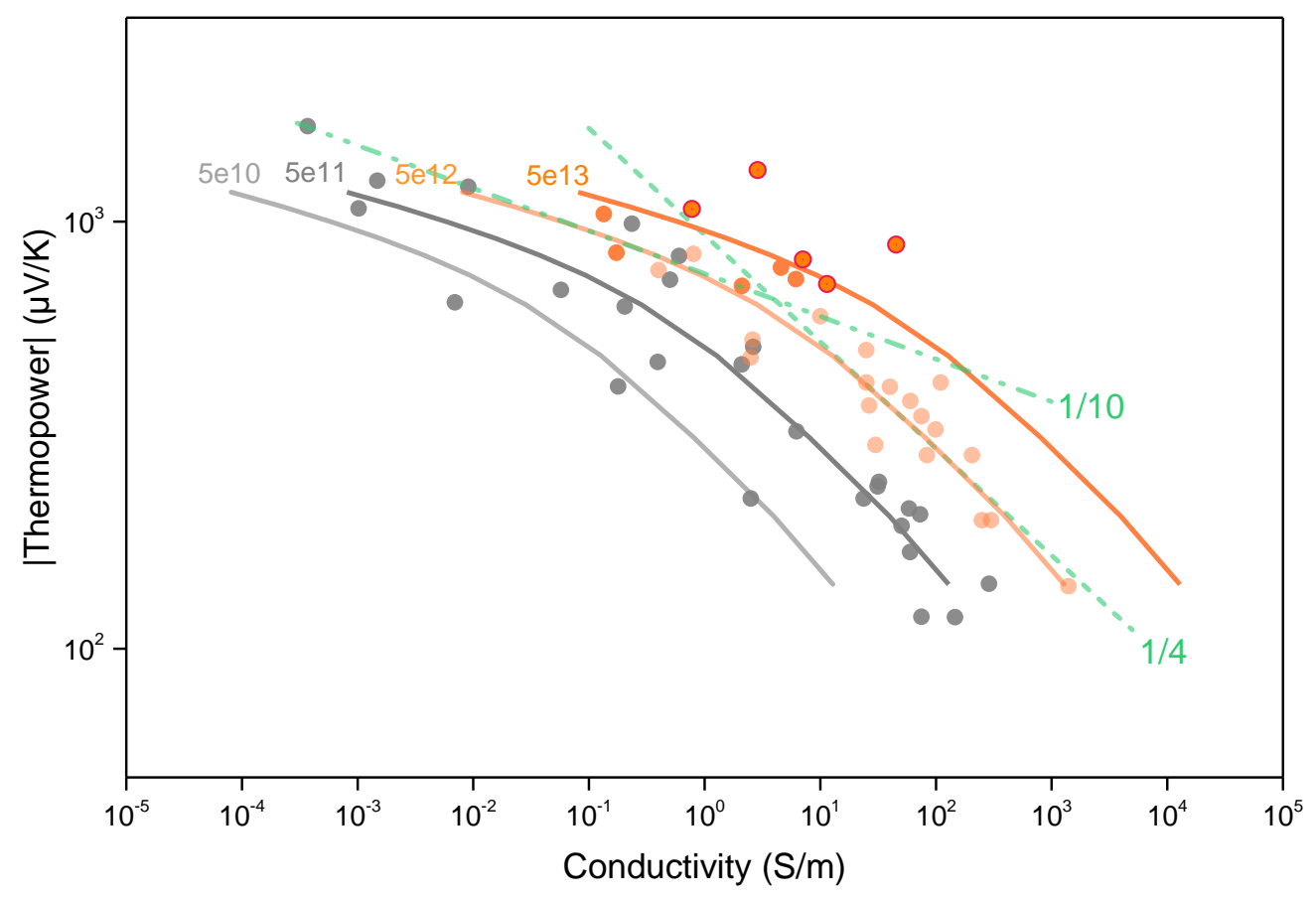

Figure 4 Thermopower vs. conductivity of doped OSC, parametric in attempt to hop frequency $v_{0}$ from $5 \mathrm{e} 10 \mathrm{~s}^{-1}$ to $5 \mathrm{e} 13 \mathrm{~s}^{-1}$. Gray and yellow dots indicate $\mathrm{p}$ - and n-type OSC, 


\section{WILEY-VCH}

respectively; light yellow data are taken from Refs. ${ }^{[1,12,15,28]}$ and dark yellow from this work; for the latter, dots w/ and w/o red perimeter are for inverse-sequential doping and bulk doping, respectively; All p-type data come from our group (Ref. ${ }^{[23]}$ ). Lines are model calculations with parameters: inter-site distance $a_{N N}=1.8 \mathrm{~nm}$; Gaussian disorder $\sigma_{\mathrm{DOS}}=0.08$ $\mathrm{eV}$; temperature $T=300 \mathrm{~K}$; inverse localization length $\alpha=5 \mathrm{e} 8 \mathrm{~nm}^{-1}$.

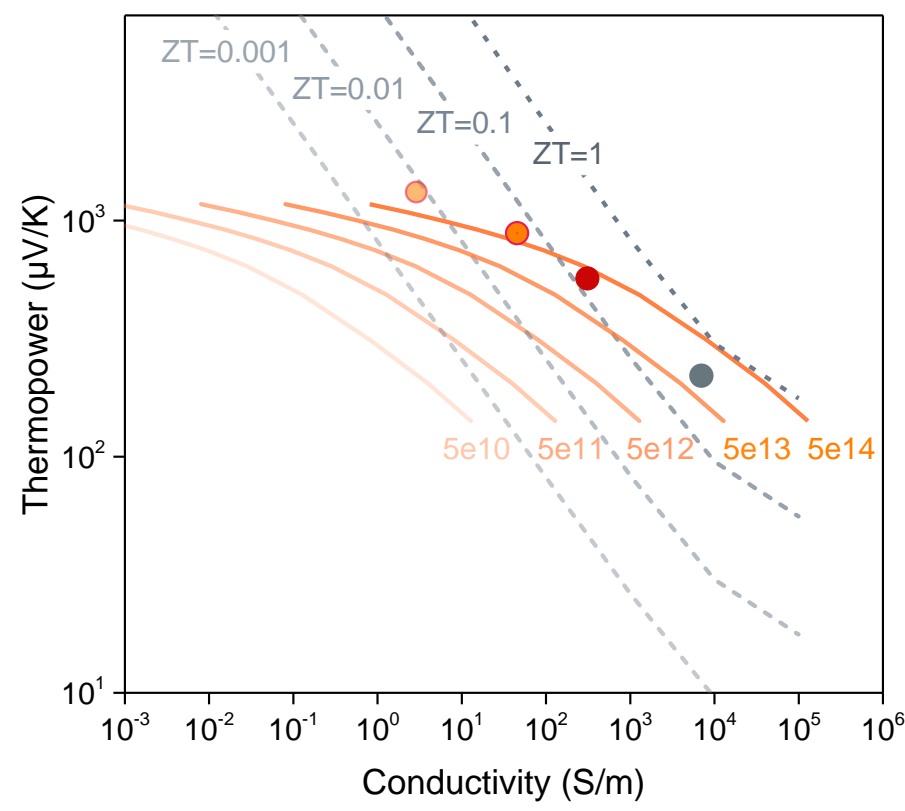

Figure 5 Contour plot of thermoelectric figure of merit ZT (dashed lines) at 300K and thermopower (solid lines) vs. conductivity parametric in the attempt to hop frequency from Figure 4. The light orange dot indicates the highest-S data point from Figure 2 and the dark orange dot the highest PF data point; the dark red dot is for (n-type) bulk N-DMBI-doped ADCV-DPPTT (Ref.[10]); the gray dot is for (p-type) PEDOT-Tos (Ref.[5]). 


\section{WILEY-VCH}

The table of contents entry should be 50-60 words long, and the first phrase should be bold. The entry should be written in the present tense and impersonal style.

Using inverse-sequential doping a very high thermoelectric power factor of $35 \mu \mathrm{Wm}^{-1} \mathrm{~K}^{-2}$ is obtained for an n-type organic semiconductor. Adding a small fraction of low-LUMO guest material can significantly increase thermopower, depending on morphology. Both pand n-type organic thermoelectrics follow a quasi-universal power law between thermopower and conductivity, with the n-type curve shifted to higher conductivities by two orders-ofmagnitude.

Keyword: Seebeck coefficient, organic thermoelectrics, power factor, n-type doping

Guangzheng Zuo, Zhaojun Li, Ergang Wang, and Martijn Kemerink*

High Seebeck coefficient and Power Factor in n-Type Organic Thermoelectrics

ToC figure $(($ Please choose one size: $55 \mathrm{~mm}$ broad $\times 50 \mathrm{~mm}$ high or $110 \mathrm{~mm}$ broad $\times 20 \mathrm{~mm}$ high. Please do not use any other dimensions))

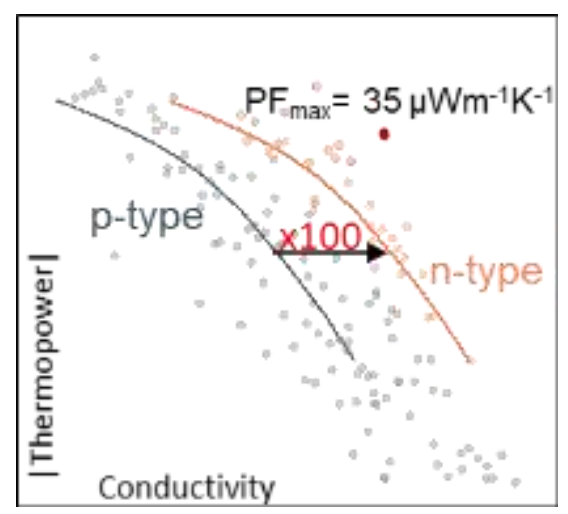




\section{WILEY-VCH}

Copyright WILEY-VCH Verlag GmbH \& Co. KGaA, 69469 Weinheim, Germany, 2017.

\section{Supporting Information}

High Seebeck coefficient and Power Factor in n-Type Organic Thermoelectrics

Guangzheng Zuo, Zhaojun Li, Ergang Wang, and Martijn Kemerink*

G. Zuo, Prof. M. Kemerink

Complex Materials and Devices, Department of Physics, Chemistry and Biology, Linköping University, SE-58183, Linköping, Sweden

E-mail: martijn.kemerink@liu.se

Z. Li, Dr. E. Wang

Department of Chemistry and Chemical Engineering, Chalmers University of Technology, SE-41296, Göteborg, Sweden 


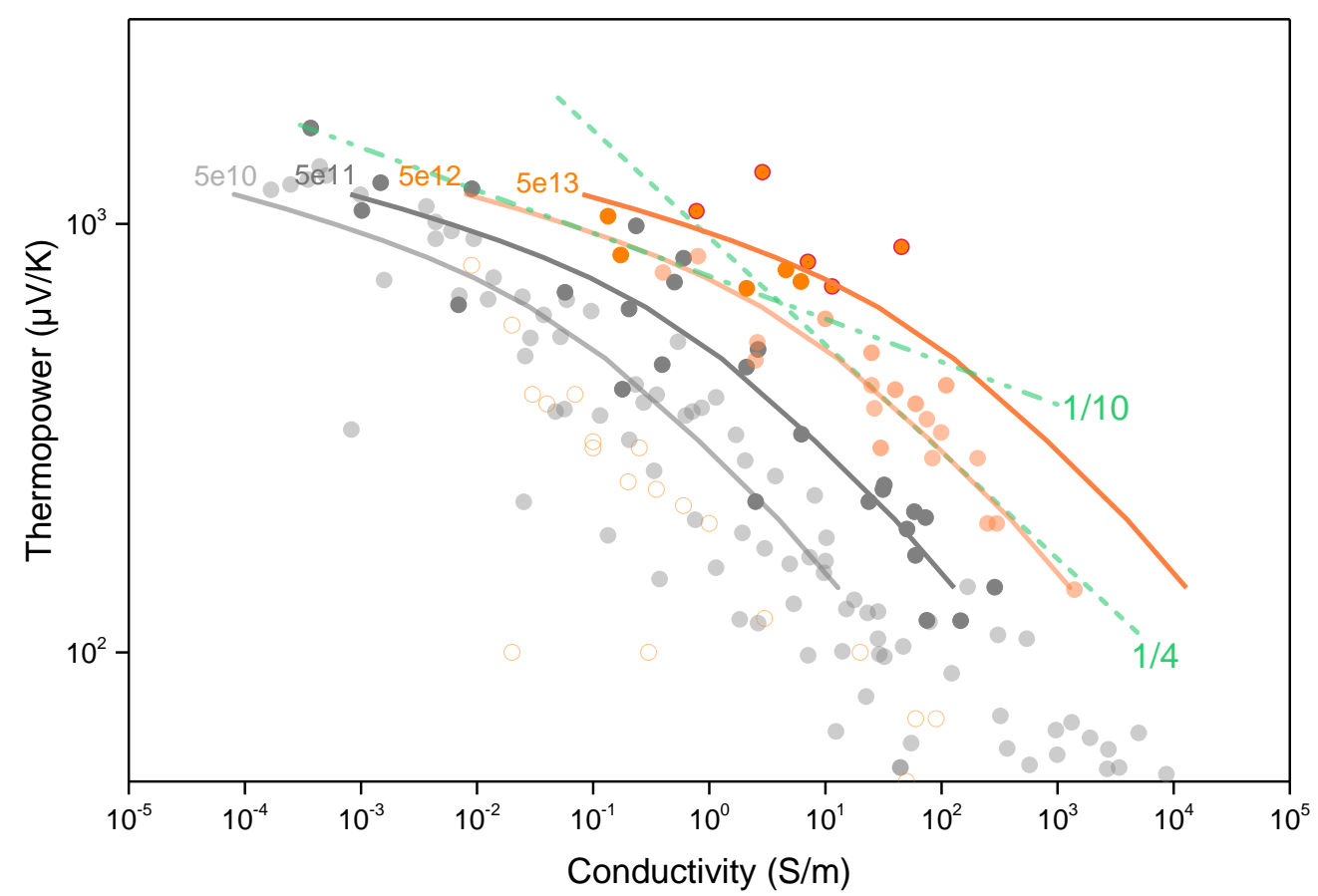

Figure S1 Extended version of Figure 4 in the main text. Here, grey dots are for p-type OSC; orange dots are for n-type OSC. Light grey dots are p-type data as assembled in $\operatorname{Ref}^{[3]}$ of the main text; dark grey data are from our own lab. The open orange symbols are n-type data from Ref. ${ }^{[16]}$. Note that the same polymer N2200 also appears in the closed light orange dataset, suggesting a problem with the TDAE dopant used. 


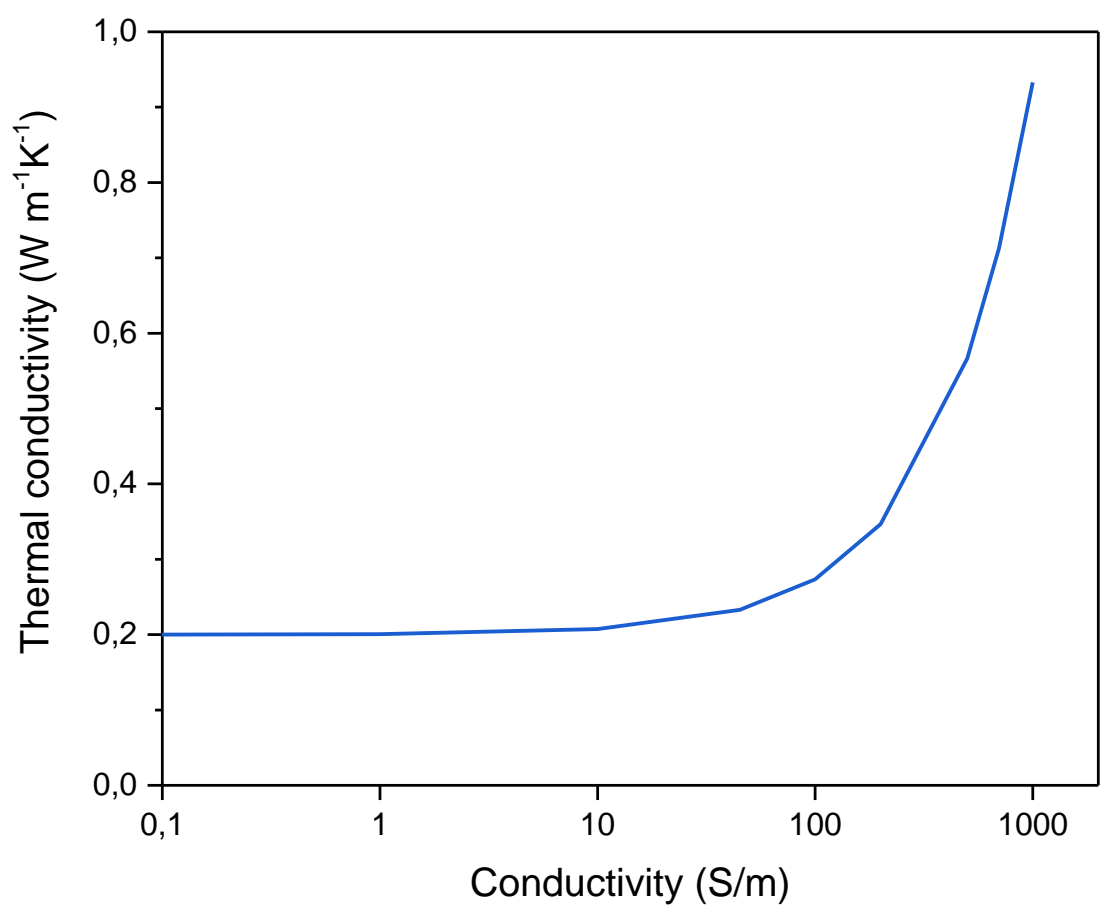

Figure S2 Thermal conductivity vs. electrical conductivity at $300 \mathrm{~K}$ as used in Figure 5 of the main text. $\kappa=\kappa_{e}+\kappa_{l} ; \kappa_{e}=\left(k_{B} / e\right)^{2} L \sigma T, L=L_{0}=\pi^{2} / 3, \kappa_{l}=0.2 \mathrm{~W} \mathrm{~m}^{-1} \mathrm{~K}^{-1}$. 


\section{WILEY-VCH}

Copyright WILEY-VCH Verlag GmbH \& Co. KGaA, 69469 Weinheim, Germany, 2017.

\section{Supporting Information}

\section{High Seebeck coefficient and Power Factor in n-Type Organic Thermoelectrics}

Guangzheng Zuo, Zhaojun Li, Ergang Wang, and Martijn Kemerink*

G. Zuo, Prof. M. Kemerink

Complex Materials and Devices, Department of Physics, Chemistry and Biology, Linköping University, SE-58183, Linköping, Sweden

E-mail: martijn.kemerink@liu.se

\section{Z. Li, Dr. E. Wang}

Department of Chemistry and Chemical Engineering, Chalmers University of Technology, SE-41296, Göteborg, Sweden 


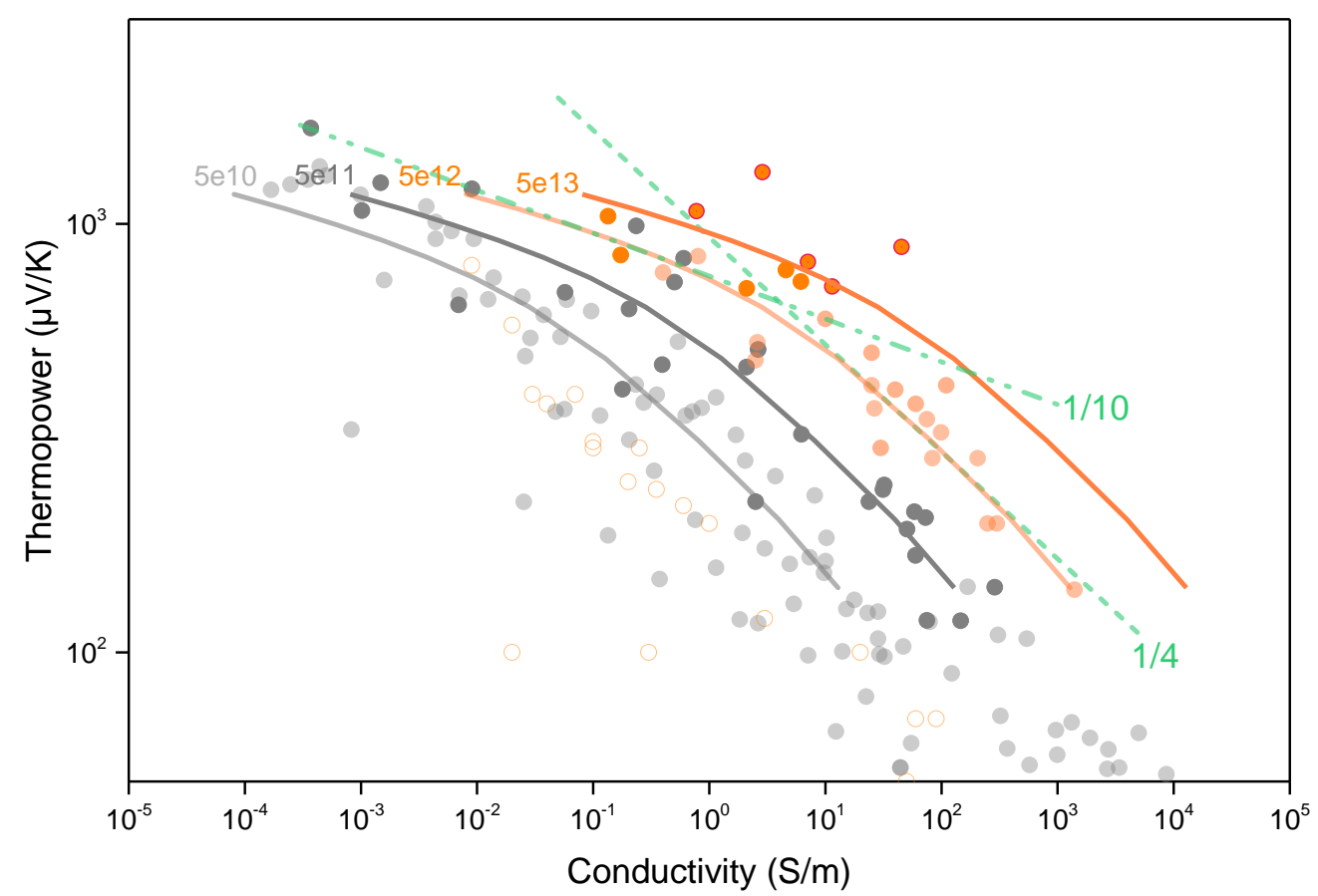

Figure S1 Extended version of Figure 4 in the main text. Here, grey dots are for p-type OSC; orange dots are for n-type OSC. Light grey dots are p-type data as assembled in $\operatorname{Ref}^{[3]}$ of the main text; dark grey data are from our own lab. The open orange symbols are n-type data from Ref. ${ }^{[16]}$. Note that the same polymer N2200 also appears in the closed light orange dataset, suggesting a problem with the TDAE dopant used. 


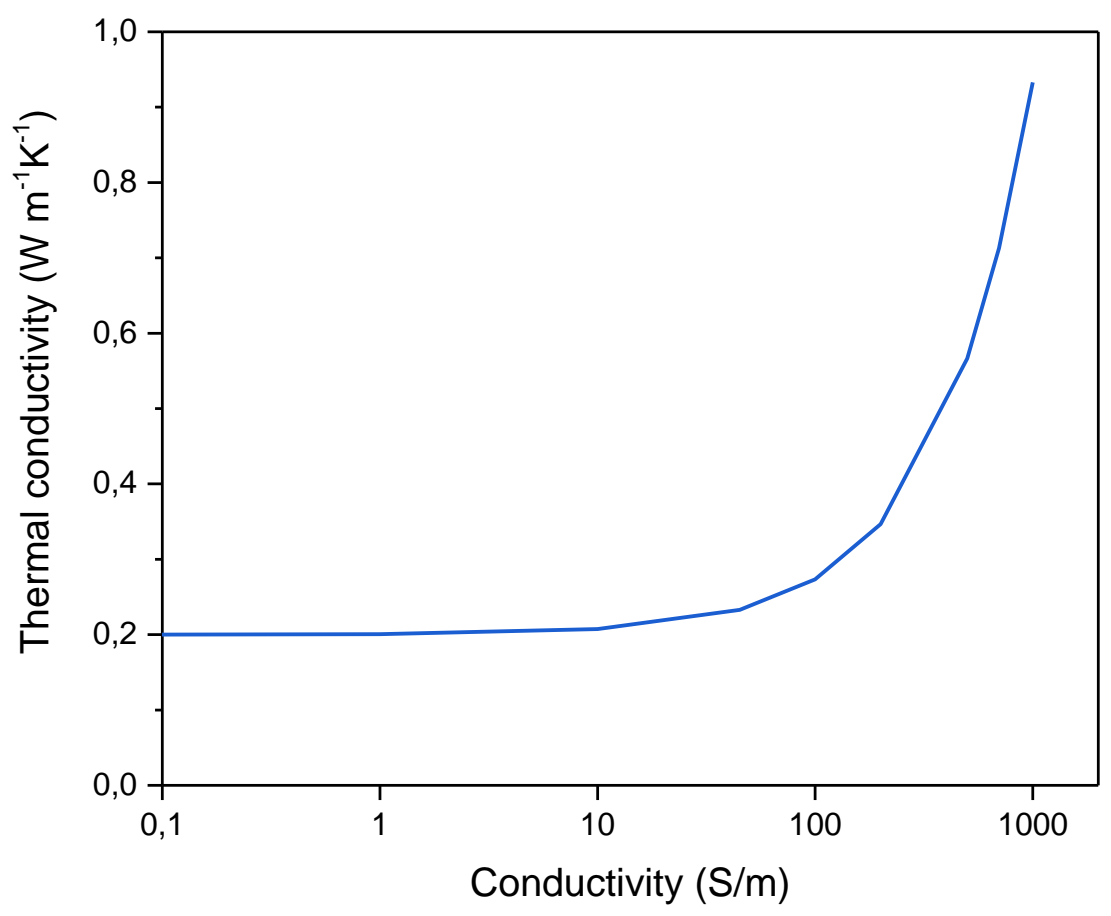

Figure S2 Thermal conductivity vs. electrical conductivity at $300 \mathrm{~K}$ as used in Figure 5 of the main text. $\kappa=\kappa_{e}+\kappa_{l} ; \kappa_{e}=\left(k_{B} / e\right)^{2} L \sigma T, L=L_{0}=\pi^{2} / 3, \kappa_{l}=0.2 \mathrm{~W} \mathrm{~m}^{-1} \mathrm{~K}^{-1}$. 